\title{
Neue Entwicklungen in der Pflegekinderhilfe
}

\section{Aktuelle Diskurse in Wissenschaft und Fachpraxis der Vollzeitpflege}

Das Aufwachsen junger Menschen außerhalb des bisherigen Elternhauses ist kein neues Phänomen. Historisch kann nachgezeichnet werden, dass Kinder und Jugendliche aufgrund des Todes ihrer Eltern oder auch durch Armuts- und andere Notlagen ihrer Familien auch in früheren Zeiten in anderen familiären wie nicht-familiären, formellen wie informellen Kontexten gelebt haben. Obwohl die Pflegekinderhilfe so auch systematisch zur Geschichte der Kinder- und Jugendhilfe gehört, wurde sie in der Fachöffentlichkeit in Deutschland über viele Jahre nur von wenigen Wissenschaftler_innen und Expert_innen diskutiert.

I nsgesamt ist festzustellen, dass die fachliche Aufmerksamkeit zur Heimerziehung im Vergleich zur Vollzeitpflege weitaus größer ist: So gibt es viel mehr Studien zu den Strukturen von stationären Einrichtungen der Kinder- und Jugendhilfe, zum Alltag der jungen Menschen und zu den Erfahrungen der darin agierenden Akteure.

Gleichwohl ist in den letzten Jahren durch Studien und Beiträge der Forschungsgruppe Pflegekinder an der Universität Siegen, des Deutschen Jugendinstituts (DJI), der

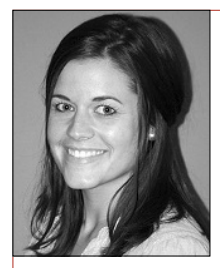

\section{Carolin Ehlke}

Hildesheim, Deutschland

*1987; Dr. phil., Studium der Erziehungswissenschaft an der Universität Hildesheim, seit 2013 wissenschaftliche Mitarbeiterin am Institut für Sozial- und Organisationspädagogik, Universität Hildesheim.

carolin.ehlke@uni-hildesheim.de

Wolfgang Schröer
Hildesheim, Deutschland
*1967; Professor an der Universität Hildesheim. Arbeitsschwer-
punkte: Kinder- und Jugendhilfe, Transnationale Soziale Arbeit.
schroeer@uni-hildesheim.de

Zusammenfassung Im Vergleich zur sogenannten Heimerziehung gibt es im Bereich der Pflegekinderhilfe bislang weniger empirische Studien, die umfassende Erkenntnisse zu den Strukturen von Vollzeitpflege und den Erfahrungen der in ihr agierenden Akteure liefern. Gleichwohl ist in den letzten Jahren eine Fachdebatte entstanden, die nachhaltig die Strukturen der Pflegekinderhilfe beeinflussen und verändern könnte. Der Schwerpunkt gibt einen Einblick in aktuelle Diskurse der Wissenschaft und Fachpraxis der Vollzeitpflege.

Schlüsselwörter Pflegekinderhilfe, Vollzeitpflege, Aktuelle Diskurse, Weiterentwicklung, Wissenschaft, Fachpraxis
Gesellschaft für innovative Sozialforschung und Sozialplanung e.V. (GISS) in Bremen, des Bundesverbands für Pflege- und Adoptivfamilien e.V. (PFAD), des Kompetenzzentrums Pflegekinder, der Stiftung zum Wohl des Pflegekindes oder des Bundesverbandes behinderter Pflegekinder e. V., um nur einige zu nennen, eine neue Fachdebatte entstanden, die nachhaltig die Infrastruktur der Pflegekinderhilfe verändern könnte. So hat die Internationale Gesellschaft für erzieherische Hilfen (IGfH) 2015 ein Dialogforum Pflegekinderhilfe - gefördert durch das BMFSFJ - ins Leben gerufen, in dem diese Entwicklungen gebündelt und Vorschläge zur Veränderung und Reform der Pflegekinderhilfe erarbeitet werden (www.dialogforum-pflegekinderhilfe.de). Zudem hat auch die Landespolitik - wie z. B. in Niedersachsen (Niedersächsischen Ministerium 2016; Kuhls et al. 2014) - die Entwicklung aufgegriffen und Empfehlungen für die Weiterentwicklung der Pflegekinderhilfe erarbeitet.

Insgesamt ist eine Zunahme der Anzahl an Kindern und Jugendlichen zu beobachten, die durch Pflegefamilien betreut werden. Damit eng zusammenhängend gibt es in einigen Regionen einen großen Bedarf an Pflegefamilien. Gleichzeitig verändern sich auch die Anforderungen und in der Pflegekinderhilfe differenzieren sich weiter unterschiedliche Angebotsformen aus. Es werden in der Fachdebatte des Weiteren intensiv die Kooperationen und Beratungsmöglichkeiten der Eltern oder die Bedeutung von sogenannten Netzwerkfamilien oder der Verwandtenpflege diskutiert oder die Verwirklichung der Rechte der jungen Menschen analysiert. Zusammenfassend betrachtet steht die Infrastruktur der Pflegekinderhilfe (Wolf 2015) in ihrer Kooperationsstruktur und in ihren unterschiedlichen sozialen Beziehungsgefügen im Vordergrund. Diese Infrastruktur für die Kinder, Jugendlichen und jungen Erwachsenen, die Pflegefamili- 
en sowie Elternschaften verlässlicher, transparenter, beteiligungsorientierter und nachhaltig unterstützend $\mathrm{zu}$ gestalten, erscheint als eine große Herausforderung. Hier ist aus Sicht des Dialogforums Pflegekinderhilfe auch die gegenwärtige Reform zum Kinder- und Jugendhilfegesetz - SGB VIII - gefragt.

Die Pflegekinderhilfe steht insgesamt im Spannungsfeld zwischen familienbezogener Privatheit und jugendhilferechtlicher Öffentlichkeit. In den Beiträgen des Schwerpunkts wird dieses Spannungsfeld immer wieder offensichtlich: Wie können die (Schutz-)Rechte, die junge Menschen haben, in Pflegefamilien verwirklicht werden? Welche Herausforderungen erleben Pflegeeltern in Verwandtenpflegeverhältnissen? Was bedeutet Qualität bzw. Professionalität in der Pflegekinderhilfe? Wie bewältigen Care Leaver den Übergang aus ihren Pflegefamilien in ein eigenverantwortliches Leben? Diesen Fragen geht der Schwerpunkt zu aktuellen Entwicklungen in der Pflegekinderhilfe nach.

In dem Beitrag von Laura Husmann und Tanja Rusack stehen die Rechte von jungen Menschen in Pflegefamilien im Vordergrund. Unter Bezug auf die Diskussionen um sogenannte Schutzkonzepte machen sie deutlich, dass es zur Verwirklichung der Rechte von jungen Menschen in der Pflegekinderhilfe Standards braucht. Im aktuell laufenden Projekt „FosterCare“ der Universität Hildesheim, der Hochschule Landshut und des Universitätsklinikums Ulm wird untersucht, wie die höchstpersönlichen Rechte junger Menschen in Pflegefamilien gestärkt und geschützt werden können. Ein zentrales Ergebnis ist, dass Schutzkonzepte in stationären Jugendhilfeeinrichtungen nicht einfach auf das familiäre Setting von Pflegeverhältnissen übertragen werden können. In dem Beitrag wird u. a. auch dargestellt, welcher gesetzlicher Änderungen es im SGB VIII bedarf, um die Absicherung von Schutzkonzepten in der Pflegekinderhilfe zu gewährleisten.

Lilli Frischmuth wirft in ihrem Beitrag einen Blick auf Verwandtenpflege. Dieser Bereich der Pflegekinderhilfe wurde historisch gesehen schon sehr lange als mehrheitlich informelle Betreuungsform genutzt. Jedoch ist bis heute - trotz der mittlerweile zunehmenden Formalisierung von Verwandtenpflege im Kontext öffentlicher Erziehung - noch viel zu wenig darüber bekannt, welche Erfahrungen die zentralen Akteure dieser Pflegeform machen und welchen Herausforderungen sie in den Pflegeverhältnissen begegnen. Lilli Frischmuth betrachtet, welche Herausforderungen Pflegeeltern aus ihrer Sicht bewältigen müssen. Ein zentrales Ergebnis findet sich in den Rollenkonflikten und -diffusionen der Pflegeeltern. Einerseits sind sie „Dienstleitende“ für das Jugendamt, andererseits sind sie rechtlich wie emotional mit den Herkunftseltern verbunden. So entsteht eine Ver- mittler_innenrolle zwischen einem öffentlichen Erziehungshilfeauftrag und der Loyalität gegenüber eigenen Familienmitgliedern. Unter der Überschrift „Transparenz verbessern und Begleitung intensivieren " führt Lilli Frischmuth verschiedene Handlungsempfehlungen für Fachkräfte in Pflegekinderdiensten auf, um die Zusammenarbeit mit Verwandtenpflegeeltern zu verbessern.

Christian Erzberger gibt in seinem Beitrag einen Einblick in die Arbeit des Dialogforums Pflegekinderhilfe. Ziel des Forums ist es, fachliche und gesetzliche Handlungsbedarfe für die Pflegekinderhilfe herauszuarbeiten und diese der Fachöffentlichkeit zur Verfügung zu stellen. Christian Erzberger fokussiert in seinen Darstellungen auf das Thema der Qualität und Qualifizierung in der Pflegekinderhilfe und erörtert dieses entlang der Triade Fachkräfte, Pflegeeltern und Eltern. Während hinsichtlich der Fachkräfte eine Qualifizierung die beruflich-fachliche Weiterentwicklung von Kenntnissen und Kompetenzen beinhaltet, sei die Begriffsbestimmung für die zwei Familiensysteme, insbesondere die Pflegefamilien, diffus. Pflegefamilien sind keine Organisationen und die Betreuung der jungen Menschen erfolgt in einem privat-familiären Rahmen. Was bedeutet hier also Qualifizierung? Christian Erzberger führt verschiedene Handlungsansätze auf, wie Qualifizierung in der Pflegekinderhilfe auf den unterschiedlichen Akteursebenen umgesetzt werden kann. Er schreibt neben der Kinderund Jugendhilfe dem Ausbildungssystem und der Politik eine besondere Verantwortung zu.

In dem Beitrag von Carolin Ehlke wird beleuchtet, wie sich der Übergang junger Menschen - Leaving Care aus den Pflegefamilien ins Erwachsenenleben gestaltet. Dieser ist für die jungen Menschen nicht selten herausfordernd und wurde sowohl in der Fachpraxis als auch in der Forschung bislang viel zu wenig beleuchtet. Carolin Ehlke hat untersucht, wie Care Leaver aus ihrer Sicht den Übergang bewältigen und von wem sie wie dabei unterstützt wurden. Ein wichtiges Ergebnis ist, dass insbesondere die sozialen Beziehungen als unterstützend wahrgenommen wurden, in denen eine gegenseitige $\mathrm{Zu}$ gehörigkeit erfahren wurde. In den Aussagen der jungen Menschen werden dabei auch Spannungsverhältnisse hinsichtlich der rechtlichen Zugehörigkeit zu der Herkunftsfamilie und der emotionalen Zugehörigkeit zur Pflegefamilie deutlich. Unterstützungsbeziehungen müssen jedoch nicht zwangsläufig familiär begründet sein. Carolin Ehlke plädiert dafür, soziale Beziehungen und Netzwerke von Care Leavern im Übergangsprozess unter dem Label eines „doing relationships“ wahrzunehmen und zu fördern. 
Funding. Open Access funding provided by Projekt DEAL.

Open Access. Dieser Artikel wird unter der Creative Commons Namensnennung 4.0 International Lizenz veröffentlicht, welche die Nutzung, Vervielfältigung, Bearbeitung, Verbreitung und Wiedergabe in jeglichem Medium und Format erlaubt, sofern Sie den/die ursprünglichen Autor(en) und die Quelle ordnungsgemäß nennen, einen Link zur Creative Commons Lizenz beifügen und angeben, ob Änderungen vorgenommen wurden.

Die in diesem Artikel enthaltenen Bilder und sonstiges Drittmaterial unterliegen ebenfalls der genannten Creative Commons Lizenz, sofern sich aus der Abbildungslegende nichts anderes ergibt. Sofern das betreffende Material nicht unter der genannten Creative Commons Lizenz steht und die betreffende Handlung nicht nach gesetzlichen Vorschriften erlaubt ist, ist für die oben aufgeführten Weiterverwendungen des Materials die Einwilligung des jeweiligen Rechteinhabers einzuholen.

Weitere Details zur Lizenz entnehmen Sie bitte der Lizenzinformation auf http://creativecommons.org/licenses/by/4.0/deed.de.

\section{Literatur}

Kuhls, A., Glaum, J., \& Schröer, W. (Hrsg.). (2014). Pflegekinderhilfe im Aufbruch. Aktuelle Entwicklungen und neue Herausforderungen in der Vollzeitpflege. Weinheim, Basel: Beltz Juventa.

Niedersächsisches Ministerium für Soziales, Gesundheit und Gleichstellung (2016). Weiterentwicklung der Vollzeitpflege. Anregungen und Empfehlungen für die Niedersächsischen Jugendämter. Bremen: Gesellschaft für innovative Sozialforschung und Sozialplanung e. V.

Wolf, K. (Hrsg.). (2015). Sozialpädagogische Pflegekinderforschung. Bad Heilbrunn: Julius Klinkhardt.

Hier steht eine Anzeige.

Springer 